\title{
REPRESENTATIONS OF LATTICES BY SETS
}

\author{
BY \\ GARRETT BIRKHOFF AND ORRIN FRINK, JR.
}

This paper deals with the representations of a general lattice $L$ by sets. After a preliminary extension of Tukey's concept of a "property of finite character" to closure properties, necessary and sufficient conditions are given for $L$ to be isomorphic with the lattice of all subalgebras of a suitable abstract algebra. It is shown that in any lattice $L$ satisfying these conditions, every element is a meet of completely meet-irreducible elements, and that this generalizes the main result of Garrett Birkhoff [3](1) on subdirect unions in universal algebra. Komatu's necessary and sufficient conditions are derived for $L$ to be isomorphic with the lattice of all ideals of another lattice $A$.

Attention is then turned to more general representations of lattices by sets of elements of any kind, and in particular by sets of dual ideals. It is first shown that meet-representations correspond to sets of dual ideals, and that isomorphic representations are furnished by all meet-irreducible, by all completely meet-irreducible, and by all principal dual ideals. In distributive lattices, meet-irreducible and prime dual ideals are the same; the representation by meet-irreducible dual ideals gives the representation theorem of Stone [2]; the representation by completely meet-irreducible dual ideals gives that of Garrett Birkhoff $[1 ; 3]$. The latter is perhaps preferable on grounds of economy; but in lattices with the disjunction property of Wallman [1], the still more economical representation by maximal dual ideals is to be preferred.

In any case, the concept of a completely irreducible ideal, which is applicable also to rings, seems worthwhile.

The paper concludes with some instructive counter-examples, and with a description of how, in the case of lattices with the descending chain condition, some of our representation schemes reduce to that introduced by A. D. Campbell [1].

\section{Part I. Lattices of SUbalgebras}

1. Closure properties of finite character. Let $A$ be any abstract algebra, in the most general sense; let $\Phi$ denote the class of all subalgebras of $A$, or, equivalently, the property of being a subalgebra. It is well known that any intersection of subalgebras is a subalgebra, and since $A$ is a subalgebra of itself, it follows that $\Phi$ is a closure property on the subsets of $A$, and is extensionally attainable in the sense of $\mathrm{E}$. H. Moore. We shall now show that if

Presented to the Society, December 31, 1947; received by the editors July 18, 1947.

(1) Numbers in brackets refer to the bibliography at the end of the paper. 
the operations of $A$ are $f$ initary $\left({ }^{2}\right)$ (that is, algebraic, rather than topological), then $\Phi$ is also "of finite character" $\left({ }^{3}\right)$, in the following general sense.

DEFINITION. A property $\Phi$ of the subsets $S$ of a set $I$ is "of finite character" if and only if, for some set $\Psi_{0}$ of finite subsets $F$ of $I$, and set $\Psi_{1}$ of ordered pairs $(G, F)$ such that $G \leqq F$ and $F \in \Psi_{0}$, it is true that $S \in \Phi$ if and only $(S \cap F, F) \in \Psi_{1}$ for all $F \in \Psi_{0}$. If no $F \in \Psi_{0}$ contains more than $n$ elements; we shall say that $\Phi$ is "of character $n$ " or less.

LEMMA 1. The property of being a subalgebra is of finite character, in any algebra with finitary operations.

Proof. A set $S$ is in $\Phi$ if and only if, for every finite subset $F=\left\{a_{0}, a_{1}\right.$, $\left.\cdots, a_{n}\right\}$ such that $\left\{a_{1}, \cdots, a_{n}\right\} \leqq S$ and $a_{0}=f_{\alpha}\left(a_{1}, \cdots, a_{n}\right)$, for some operation $f_{\alpha}$ of the algebra $A$ we have $a_{0} \in S$. Incidentally, we see in this way that an $n$-ary operation is of character $n+1$.

THEOREM 1. For a closure property $\Phi$ on a set $I$, the following three conditions are equivalent:

(i) For some determination of I as an abstract algebra with finitary operations, $S \in \Phi$ means that $S$ is a subalgebra;

(ii) The property $\Phi$ is of finite character;

(iii) The set-union $X$ of any directed family $\left({ }^{4}\right)$ of sets $X_{\alpha} \in \Phi$ itself belongs to $\Phi$.

Proof. By Lemma 1, (i) implies (ii). Again, let (ii) hold, and let $X$ be the set-union of any directed family of $X_{\alpha}$ in $\Phi$. If $F$ is any finite subset of $I$, we can enumerate the elements of $X \cap F$ as $a_{1}, \cdots, a_{n}$; for some $\alpha(1), \cdots, \alpha(n)$, clearly $a_{i} \in X_{\alpha(i)}$. Any common successor $X_{\gamma}$ of $X_{\alpha(1)}, \cdots, X_{\alpha(n)}$ will contain $X \cap F$; but $X_{\gamma} \leqq X$, hence $(X \cap F, F)=\left(X_{\alpha} \cap F, F\right) \in \Psi_{1}$ by hypothesis. Therefore $X$ is closed, and (ii) implies (iii). Finally, suppose that (iii) is satisfied. For each determination $\alpha$ of a finite $F$ and an $a$ in $\bar{F}-F$, we define an $n$-ary operation $f_{\alpha}$, as follows:

$$
\begin{array}{ll}
f_{\alpha}\left(x_{1}, \cdots, x_{n}\right)=a, & \text { if every } x_{i} \in F, \\
f_{\alpha}\left(x_{1}, \cdots, x_{n}\right)=x_{1}, & \text { if some } x_{i} \in F .
\end{array}
$$

Here $n$ is the number of elements in $F$. This definition makes $I$ into an abstract algebra $A$ with finitary operations. By (1) and the definition of subalgebra, $X$ is a subalgebra if and only if for every finite subset $F_{\alpha}$ of $X$, we have $\bar{F}_{\alpha} \leqq X$. But if $X \in \Phi$, clearly $\bar{F}_{\alpha} \leqq \bar{X}=X$; hence $X$ is a subalgebra. Con-

(2) The terminology is that of Garrett Birkhoff, Lattice theory, chap. II, and [3] in universal algebra.

${ }^{(3)}$ The present definition includes that of J. W. Tukey, Convergence and uniformity in topology, Princeton, 1940. Tukey's definition is well adapted to independence properties, but not to closure properties.

(4) In the sense of Moore-Smith: given $X_{\alpha}$ and $X_{\beta}$, there exists an $X_{\gamma}$ in the family, which contains both $X_{\alpha}$ and $X_{\beta}$. 
versely, suppose $X$ is a subalgebra. The $F_{\alpha} \leqq X$ form a directed family; hence so do the $\bar{F}_{\alpha} \leqq X$, also partially ordered by set-inclusion. Hence, by (iii), $\cup \bar{F}_{\alpha} \in \Phi$. But $X=\bigcup F_{\alpha} \leqq U \bar{F}_{\alpha} \leqq X$, so that $X \in \Phi$, completing the identification of $\Phi$ with the property of being a subalgebra. Therefore (iii) $\rightarrow$ (i), completing the proof.

2. Lattice-theoretic implications. We now determine the implications of the preceding section for the abstract lattice $L$ of all subalgebras of an abstract algebra $A$, partially ordered by set-inclusion. It is well known that $L$ is complete if and only if, for some class $I$ and closure property $\Phi$ on $I, L$ is isomorphic with the lattice of all subsets of $I$ in $\Phi$.

We now ask, what does it mean for $L$ that $A$ has finitary operations-or equivalently, by Theorem 1 , that $\Phi$ is of finite character? We shall show that this is equivalent to two further restrictions on $L$.

LEMMA 2. In the complete lattice $L$ of all subalgebras of any abstract algebra with finitary operations,

$$
X_{\alpha} \uparrow X \quad \text { and } \quad Y_{\beta} \uparrow Y \quad \text { imply } \quad\left(X_{\alpha} \cap Y_{\beta}\right) \uparrow X \cap Y,
$$

for any directed sets.

Proof. By (ii) of Theorem 1, $X$ and $Y$ are the set-unions of the $X_{\alpha}$ and $Y_{\beta}$ respectively; moreover $\cap$ means set-intersection, and (2) is known to hold for set-unions and intersections.

Incidentally, (2) follows from the apparently weaker special case:

$$
X_{\alpha} \uparrow X \text { implies } X_{\alpha} \cap Y \uparrow X \cap Y \text {. }
$$

For, assuming $\left(2^{\prime}\right)$, we see that $\mathrm{U}\left(X_{\alpha} \cap Y_{\beta}\right)$ contains every $X \cap Y_{\beta}$, hence it contains $\mathrm{U}\left(X \cap Y_{\beta}\right)=X \cap Y$, using $\left(2^{\prime}\right)$ again.

Incidentally also, (2) and $\left(2^{\prime}\right)$ hold for finite sequences in any lattice. Thus, for two-element sequences, $\left(2^{\prime}\right)$ reduces to

$$
\text { if } x \leqq z \text {, then }(x \cup z) \cap y=(x \cap y) \cup(z \cap y) \text {, }
$$

which formally resembles the modular identity $\left({ }^{5}\right)$. Condition (2) asserts essentially that meets are continuous in the order topology.

The second restriction on $L$ is also of a topological nature, and is suggested by a consideration of the principal subalgebras $S=\{s\}$ of $A$, each generated by a single element $s$. Such subalgebras are seen to be $\uparrow$-inaccessible (or inaccessible from below), in the sense that if, for some directed set $\left\{X_{\alpha}\right\}$, we have $X_{\alpha} \uparrow S$, then some individual $X_{\alpha}$ equals $S$. For, since $S$ is the set-union of the $X_{\alpha}$, by (iii) some $X_{\alpha}$ contains $s$, hence $\xi=S$. But every $X_{\alpha} \leqq S$; hence this $X_{\alpha}=S$.

(5) Thus the modular identity is equivalent to the condition that each one of the six conceivable distributive equalities between a triple of elements implies all five others. 
Caution: Being $\uparrow$-inaccessible is equivalent to being finitely generated; not every $\uparrow$-inaccessible subalgebra need be principal.

Since every subalgebra is (obviously) the join of the principal subalgebras which it contains, we infer the following lemma.

LEMma 3. In the lattice L of Lemma 2, every element is a join of $\uparrow$-inaccessible elements.

We are now in a position to characterize abstractly those lattices which are isomorphic with the lattice of all subalgebras of some abstract algebra with finitary operations.

THEOREM 2. An abstract lattice $L$ is isomorphic with the lattice of all subalgebras of a suitable abstract algebra $A$ with finitary operations, if and only if it is complete, satisfies (2), and satisfies the condition that every element is a join of $\uparrow$-inaccessible elements.

Proof. By Lemmas 2 and 3, we need only prove the sufficiency of our conditions. Accordingly, let $L$ be given as described. We let the elements of $A$ be the $\uparrow$-inaccessible elements of $L$. We call a subset $S$ of $A$ "closed" if and only if $a, b \in S, x \in A$ and $x \leqq a \cup b$ in $L$ imply $x \in S$. This is a closure property of character three; hence by Theorem 1 , the lattice $M$ of all "closed" subsets is a subalgebra lattice of the desired type. Hence it remains only to show that $M$ and $L$ are isomorphic; this we now do.

Evidently, if $x \in L$, the set $S(x)$ of all $a \leqq x$ is closed. Conversely, let $S \in M$ be any closed subset of $A$; define $x(S)=\bigcup a_{\alpha}$ to be the join in $L$ of the $a_{\alpha} \in S$. Consider the directed set of all joins $S_{\phi}$ of finite sets $\phi$ of $a \in S$; in $L, S_{\phi} \uparrow x(S)$ by construction. Hence, by $\left(2^{\prime}\right), a \leqq x(S)$ implies $a=a \bigcap x(S)=\mathrm{U}\left(a \cap S_{\phi}\right)$. Hence, $a$ being $\uparrow$-inaccessible, some $a \cap S_{\phi}=a$, that is, some $S_{\phi}$ contains $a$. In other words, $a \leqq a_{1} \cup \cdots \cup a_{n}$ for some finite subset $\phi=\left\{a_{1}, \cdots, a_{n}\right\}$ of $S$. Since $S$ is closed, it follows by finite induction that $a \in S$. That is, $a \leqq x(S)$ implies $a \in S$; but the converse is trivial, hence $S(x(S))=S$. Finally, since every $x$ is (by hypothesis) the join of join-inaccessible elements, $x(S(x))=x$. Hence our isotone (that is, order preserving) correspondence is one-to-one, and $M$ and $L$ are isomorphic.

It is a corollary that we can strengthen (2) to

$$
X_{\beta}^{\sigma} \uparrow X^{\sigma} \text { implies } \sup _{\beta(\sigma)}\left\{\bigcap_{\sigma} X_{\beta(\sigma)}^{\sigma}\right\}=\bigcap X^{\sigma} .
$$

3. Atomicity; completely irreducible elements. We now show that the lattices considered in Theorem 2 satisfy also a kind of atomicity condition. Let $L$ satisfy the conditions of Theorem 2 , and suppose $x>y$ in $L$. By hypothesis, we can choose a $\uparrow$-inaccessible element $z$ such that $z>y$ and $z \leqq x$. If $C$ is a maximal chain of elements $z_{\tau}<z$, than $c=U z_{\tau}<z$ by $\uparrow$-inaccessibility. Since $C$ is maximal, however, $c<t<z$ is impossible; hence $z$ covers $c$. 
Thus we have:

Lemma 4. If $x>y$ in a lattice satisfying the conditions of Theorem 2 , then there exists a pair $z, c$, such that $x \geqq z>c \geqq y$, and $z$ covers $c$.

This is a weak atomicity condition (see Lattice theory, p. 13). If $L$ is a Boolean algebra, $z \cap c^{\prime}$ is a point contained in $x$. Hence (Lattice theory, p. 95.) $L$ is an atomic Boolean algebra. We conclude $\left(^{6}\right)$ :

THEOR EM 3. If the subalgebras of an abstract algebra with finitary operations form a Boolean algebra $B$, then $B$ is isomorphic with the atomic Boolean algebra of all subsets of a suitable class.

More generally, let $L$ be any complete lattice satisfying (2), and let $a$ be any $\uparrow$-inaccessible element of $L$. Let $C=\left\{x_{\alpha}\right\}$ be any maximal chain such that no $x_{\alpha} \in C$ contains $a$; such a $C$ exists by Zorn's lemma. Form $c=\bigcup x_{\alpha}$; since every $x_{\alpha} \cap a<a$, we have by (2), $c \cap a=\mathrm{U}\left(x_{\alpha} \cap a\right)<a$, and so it is false that $c \geqq a$. But $C$ is maximal; hence $c \in C$, which proves the following lemma.

Lemma 5. Every maximal chain $C$ of elements, none of which contains a, has a largest element $c$, if $a$ is an $\uparrow$-inaccessible element of a complete lattice.

Next, suppose $x>c$. Since $c \geqq x_{\alpha}$ for all $x_{\alpha} \in C$, we still have a chain if we add $x$ to $C$. Since $C$ was maximal subject to the condition that $x_{\alpha} \geqq a$ is false, it follows that $x \geqq a$. Hence $x \geqq c \cup a$. But $c \cup a>a$. We conclude that, among all elements $t>c$, there is a least one, namely $c \cup a$.

Lemma 6. In any complete lattice $L$, the set $T$ of elements $t_{\alpha}>c$ has a least element $m$, if and only if $c$ is completely meet-irreducible, in the sense that $c=\cap y_{\beta}$ implies that some $y_{\beta}=c$.

Proof. If $c$ is completely meet-irreducible, then $\cap t_{\alpha} \neq c$ by hypothesis; but $\cap t_{\alpha} \geqq c$; hence $m=\cap t_{\alpha}>c$ is a least element of $T$. Conversely, if such a least element $m$ exists, and $c=\bigcap y_{\beta}$, then all $y_{\beta} \geqq c$. But not all $y_{\beta}>c$, or $\cap y_{\beta} \geqq m$; hence some $y_{\beta}=c$.

REMARK. Complete meet-irreducibility thus implies, but is in general a stronger condition than, the dual of $\uparrow$-inaccessibility.

Combining the preceeding results, we get the following theorem.

Theorem 4. Let $a$ be any $\uparrow$-inaccessible element of a complete lattice $L$ satisfying (2).Then every maximal chain $C$ of elements $x$ of $L$ such that $x \geqq a$ is false has a largest element $c$. Moreover, $c$ is completely meet-irreducible; $a \cup c$ is the smallest element properly containing $c$.

${ }^{(6)}$ A related result was recently communicated to us orally by A. Tarski. The results of \$\$1-2 were described at the Princeton Bicentennial Conference on Mathematics, in December, 1946. 
Conversely, let $c$ be completely meet-irreducible, and let $m$ be the smallest containing element, which exists by Lemma 6 . If $m$ is a join of $\uparrow$-inaccessible elements $a_{\alpha}$, then there exists an $a_{\alpha} \leqq m$, such that $a_{\alpha} \leqq c$ is false. Clearly $c$ is maximal subject to the condition of not containing $a_{\alpha}$. We conclude:

TheOREM 5. A subalgebra of an abstract algebra $A$ with finitary operations is completely meet-irreducible in the lattice of subalgebras of $A$, if and only if it is maximal subject to failure to contain a $\uparrow$-inaccessible subalgebra.

4. Applications to congruence relations. Similar results hold for the lattice $H$ of all congruence relations $\theta$ on an abstract algebra $A$. Each such $\theta$ may be described by a subset $S(\theta)$ of the set $A \times A$ of all ordered couples $(a, b)$ of elements $a, b$ in $A$ in the usual way: $(a, b) \in S(\theta)$ means that $a \equiv b(\bmod \theta)$.

Moreover, being a congruence relation is a closure property, since the reflexive, symmetric, transitive, and substitution laws on relations $\left({ }^{7}\right)$ are each extensionally attainable. Further, the conditions that $\theta$ be reflexive, symmetric, and transitive are of character three or less; if $A$ has finitary operations, the substitution property is also of finite character. We conclude, by Theorems 1 and 2:

THEOREM 6. The lattice $H$ of all congruence relations on any abstract algebra with finitary operations satisfies the conditions of Theorem 2, and is isomorphic with the lattice of all subalgebras of some other abstract algebra $B$.

We do not know whether the converse is true. The most promising tool to attack this question seems to be Whitman's theorem that any lattice is isomorphic with a sublattice of the lattice of all partitions of a suitable aggregate, combined with the fact that any $H$ as defined above is also such a sublattice.

THEOREM 7. In any lattice $L$ satisfying the conditions of Theorem 2, every element $a$ is a meet of completely meet-irreducible elements $\left(^{8}\right)$.

Proof. Let $m=\bigcap c_{\gamma}$ be the meet of the completely meet-irreducible elements $c_{\gamma} \geqq a$. Unless $m=a$, clearly $m>a$, and some $\uparrow$-inaccessible element $b$ satisfies $b \leqq m$, but not $b \leqq a$. Form a maximal chain $D$ of elements $d_{\delta}$ such that $d_{\delta}$ includes $a$ but not $b$. Let $d$ be the largest element of $D$; by Theorem $4, d$ is completely meet-irreducible, $d \geqq a$ holds, but $d \geqq b$ does not. Hence $d$ is a $c_{\gamma}$. But this implies $d \geqq m \geqq b$, which is a contradiction. Hence $m=a$, which was to be proved.

5. Applications to lattice ideals and dual ideals. In any lattice $A$, an ideal is a "subalgebra" with respect to the binary operation $\cup$ and the unary

(7) These define congruence relations; see for example Birkhoff and MacLane, Survey of modern algebra, chap. VI, \$14.

(8) This generalizes the main result of Garrett Birkhoff, Subdirect unions in universal algebra, Bull. Amer. Math. Soc. vol. 50 (1944) pp. 764-768. 
operations $f_{a}: f_{a}(x)=x \cap a$. Hence the conclusions of Theorems 2-5 and 7 apply. Also, by Lemma 6, every maximal ideal $M$ is completely meet-irreducible.

Theorem 8. An ideal $J$ of a lattice $A$ is principal if and only if it is an $\uparrow$-inaccessible element of the lattice $L$ of ideals of $A$.

Proof. The "only if" is covered by the proof of Lemma 3 ; the "if" may be proved as follows. The principal ideals $T_{\alpha} \leqq X$ form a sublattice of $L$, since $\{t\} \cup\{u\}=\{t \cup u\}$; hence they form a directed set. Moreover clearly U $T_{\alpha}=X$. But unless $X$ is principal, every $T_{\alpha}<X$, and so $X$ is not $\uparrow$-inaccessible, completing the proof. Theorem 8 is due to A. Komatu, Proc. Imp. Acad. Tokyo vol. 19 (1943) pp. 119-124.

Corollary 1. An ideal of a lattice $A$ is completely meet-irreducible if and only if it is maximal subject to failure to contain some element of $A$.

A second corollary is the fact that the $\uparrow$-inaccessible elements of $L$ form a sublattice of $L$. Conversely, if the $\uparrow$-inaccessible elements of any lattice which satisfies the conditions of Theorem 2 form a sublattice, the construction given in the proof of Theorem 2 makes $S$ "closed" if and only if it is an ideal. We conclude:

THEOREM 9. An abstract lattice $L$ is isomorphic with the lattice of all ideals of a suitable lattice $A$ if and only if it satisfies the conditions of Theorem 2, and further the condition that the $\uparrow$-inaccessible elements of $L$ form a sublattice.

The lattice $A$ is, of course, just this sublattice of $\uparrow$-inaccessible elements. Note that if $L$ satisfies the ascending chain condition, all ideals are principal and the lattice $A$ is $L$ itself.

The preceding results apply equally to dual ideals, since a dual ideal of $A$ is simply an ideal in the dual of $A$. It is dual ideals which are important in the general theory of the representation of lattices by sets, to which we now turn our attention.

\section{PART II. RePresentations BY SETS OF DUAL IDEALS}

6. Dual ideals and meet-representations. We first state some definitions concerning lattice ideals and representations of lattices. The notation and terminology are those of G. Birkhoff [2].

A dual ideal of a lattice $L$ is a set $D$ of elements of $L$ such that $D$ contains the meet $x \cap y$ of two elements of $L$ if and only if it contains both $x$ and $y$.

A dual ideal which is not the entire lattice $L$ is said to be prime if it contains either $x$ or $y$ whenever it contains the join $x \cup y$.

A dual ideal is said to be completely prime if whenever it contains the join $U x_{a}$ of an arbitrary set $\left\{x_{a}\right\}$ of elements, it contains at least one element $x_{a}$ of the set. 
A dual ideal $D$ is said to be meet-irreducible if it is not the set-intersection of two dual ideals each distinct from $D$.

The concept of a completely meet-irreducible ideal or dual ideal, like that of a completely meet-irreducible element of a lattice, has already been discussed in $\$ \S 3$ and 5 (see Lemma 6 , and Corollary 1 to Theorem 8). A dual ideal is completely meet-irreducible if it is not the set-intersection of any number of dual ideals distinct from itself. Meet-irreducible here means intersection-irreducible. If the lattice order relation were to be reversed in the lattice of all dual ideals, as is sometimes done (see Dilworth [1] and Frink $[2])$, then intersections of dual ideals would correspond to joins rather than meets, and the term join-irreducible would be in order.

A meet-representation of a lattice $L$ is a correspondence $x \rightarrow R(x)$ between the elements $x$ of $L$ and certain sets $R(x)$, which sends lattice meets into setintersections; that is, $R(x \cap y)$ is the set-intersection of $R(x)$ and $R(y)$.

It can be seen that a meet-representation preserves also the lattice order relation, in the sense that $x \leqq y$ implies that $R(x)$ is a subset of $R(y)$. If the correspondence defining a meet-representation is one-to-one, it is said to define an isomorphic meet-representation. In this case $R(x)=R(y)$ implies that $x=y$, whence $x<y$ implies that $R(x)$ is a proper subset of $R(y)$.

In considering meet-representations of a lattice $L$ by sets of objects of some sort, there is no loss of generality if we confine attention to sets of dual ideals. For any "object" $A$ which is an element of a representative set $R(x)$ determines a dual ideal, namely the collection $D$ of all elements $x$ of $L$ such that $A$ is a member of $R(x)$. That $D$ is a dual ideal follows from the definition of dual ideal and from the fact that we have a meet-representation. If two "objects" $A$ and $B$ determine the same dual ideal in this way, then one of them is superfluous.

Conversely, it will now be shown that any collection of dual ideals of a lattice $L$ determines a meet-representation of $L$.

Theorem 10. Any collection $K$ of dual ideals of a lattice $L$ determines a meet-representation of $L$. The representation is isomorphic if and only if for every pair $x, y$ of distinct elements of $L$ there exists a dual ideal in $K$ containing one element of the pair but not the other.

Proof. Given the collection $K$, we assign to each lattice element $x$ the set $R(x)$ consisting of all dual ideals which are in $K$ and which contain the element $x$. Consider now the representative sets $R(x), R(y)$, and $R(x \cap y)$. We have a meet-representation if $R(x \cap y)$ is always the set-intersection of $R(x)$ and $R(y)$. Now note that a dual ideal $D$ is a member of $R(x)$ if and only if $D$ is a member of $K$ and $x$ is a member of $D$. Since $D$ is a dual ideal, $x \cap y$ is in $D$ if and only if $x$ and $y$ are in $D$. Hence if $D$ is in $K$, then $D$ is in $R(x \cap y)$ if and only if $D$ is in both $R(x)$ and $R(y)$. But this means that $R(x \cap y)$ is the set-intersection of $R(x)$ and $R(y)$, which was to be proved. 
The meet-representation just defined is isomorphic provided $R(x)$ and $R(y)$ are distinct whenever $x$ and $y$ are distinct. This clearly requires that the collection $K$ contain at least one dual ideal $D$ containing one of the pair $x, y$ but not the other. This completes the proof.

Theorem 10 immediately provides different representations which are obtainable by special choice of the collection $K$. The two most obvious choices for $K$ are the collection of all dual ideals, and the collection of all principal dual ideals. Since distinct elements determine distinct principal ideals, we have the following corollary.

COROLlARY. Isomorphic meet-representations of any lattice $L$ are obtained by assigning to each element $x$ of $L$ the set of all dual ideals containing $x$, or the set of all principal dual ideals containing $x$.

It will be noted later (Theorem 16) that the representation by principal dual ideals preserves not merely finite meets, but meets of arbitrarily many elements.

7. Representations by irreducible and completely irreducible ideals. It is desirable that the collection $K$ which determines a representation should contain as few elements as possible, being just large enough to discriminate between distinct elements of the lattice. We now show that $K$ may be taken to be the collection of all completely meet-irreducible dual ideals of the lattice.

TheOREM 11. An isomorphic meet-representation of any lattice $L$ is obtained by assigning to each element $x$ of $L$ the set of all completely meet-irreducible dual ideals of $L$ which contain $x$.

Proof. By Theorem 10, it is sufficient to show that if $x$ and $y$ are distinct elements of $L$, there exists a completely meet-irreducible dual ideal $D$ containing one but not the other as an element. Since $x$ and $y$ are distinct, then either $x$ is not a member of the principal dual ideal $\{y\}$, or $y$ is not a member of the principal dual ideal $\{x\}$. Suppose the latter; then by Zorn's lemma $\{x\}$ can be extended to a dual ideal $D$ which (1) does not contain $y$, and (2) is maximal with respect to the property of not containing $y$. Hence by Corollary 1 to Theorem $8, D$ is completely meet-irreducible. Also, it contains $x$ but not $y$. Likewise if $x$ is not a member of the principal ideal $\{y\}$, then a completely meet-irreducible dual ideal exists containing $y$ but not $x$. Hence by Theorem 10 the representation is isomorphic. This completes the proof.

Since any completely meet-irreducible dual ideal is also meet-irreducible, we have the following corollary.

COROLlaRy. An isomorphic meet-representation of any lattice $L$ is obtained by assigning to each element $x$ of $L$ the set of all meet-irreducible dual ideals of $L$ which contain $x$. 
We now wish to show the connection of the preceding results with other well known results on the representation of particular types of lattice by sets. In the first place, note that while the two preceding representations of Theorem 11 and its corollary are equivalent for finite lattices, they are not equivalent in general. Thus, consider the distributive lattice of all real numbers with the usual order relation. Completely meet-irreducible dual ideals consist of open semi-infinite intervals $(x, \infty)$. The merely meet-irreducible dual ideals include also the closed semi-infinite intervals $[x, \infty)$. The latter are principal dual ideals.

8. Prime ideals and distributive lattices. Theorem 11 was the result of an attempt to extend to the general lattice, as far as may be possible, the well known results of Garrett Birkhoff $[1,2]$ and Stone [2] concerning the isomorphic representation of the general distributive lattice as a ring of sets of prime ideals. We now examine the relation between this representation of distributive lattices by sets of prime ideals and the representations of Theorem 11 and its corollary by means of completely meet-irreducible and meet-irreducible dual ideals. It will be shown that in a distributive lattice, prime ideals and irreducible ideals are identical. Hence the representation of a distributive lattice $L$ given by the corollary of Theorem 11 coincides with the Stone representation by prime ideals, while the representation of $L$ by means of completely meet-irreducible dual ideals given by Theorem 11 provides the representation of Birkhoff $[1 ; 3]$, which is perhaps to be preferred on grounds of economy.

It should be noted that there is a close connection between the notion of a prime dual ideal and the dual notion of a prime ideal. In fact, prime ideals and prime dual ideals are complements of each other. Hence any representation by prime dual ideals determines a representation by prime ideals, and conversely.

THEOREM 12. In any lattice, prime dual ideals are meet-irreducible. In a distributive lattice, a dual ideal is prime if and only if it is meet-irreducible.

Proof. Suppose the dual ideal $D$ is prime but not meet-irreducible. Then $D$ is the set-intersection of two dual ideals $A$ and $B$ both distinct from $D$. Therefore $A$ contains an element $a$ not in $D$, and $B$ contains an element $b$ not in $D$. The join $a \cup b$ is in both $A$ and $B$, and hence in $D$. Since $D$ is prime, either $a$ or $b$ must be in $D$, which is a contradiction. This proves the first part of the theorem.

Now suppose the lattice $L$ is distributive, and the dual ideal $D$ is meetirreducible. To prove that $D$ is prime, suppose $a \cup b$ is in $D$, but neither $a$ nor $b$ is in $D$. Consider the two dual ideals $D \cup a$ and $D \cup b$, consisting of all elements $x$ such that $x \geqq d \cap a$ and $x \geqq d \cap b$ respectively, where $d$ is any element of $D$. Then both $D \cup a$ and $D \cup b$ are distinct from $D$. We shall now show that the set-intersection of $D \cup a$ and $D \cup b$ is $D$. Clearly every element of $D$ 
is in this intersection. Conversely, suppose that $x$ is any element of the intersection. Then $x \geqq d \cap a$ and $x \geqq d^{\prime} \cap b$, where $d$ and $d^{\prime}$ are elements of $D$. Then $x \geqq d \cap d^{\prime} \cap a$ and $x \geqq d \cap d^{\prime} \cap b$, whence $x \geqq\left(d \cap d^{\prime} \cap a\right) \cup\left(d \cap d^{\prime} \cap b\right)$. There fore, by the distributive law, $x \geqq\left(d \cap d^{\prime}\right) \cap(a \cup b)$. But $\left(d \cap d^{\prime}\right) \cap(a \cup b)$ is an element of $D$, and hence $x$ is an element of $D$. This shows that $D$ is the setintersection of $D \cup a$ and $D \cup b$. But this involves a contradiction, since $D$ was assumed to be meet-irreducible. Hence $D$ is prime. This completes the proof.

It follows from Theorem 12 that the representation of distributive lattices by sets of meet-irreducible dual ideals provided by the corollary to Theorem 11 is identical with the Birkhoff-Stone representation by prime ideals (or equivalently, by prime dual ideals) already referred to.

A representation of a lattice by sets is said to be a join-representation if it preserves joins; that is, if $R(x \cup y)$ is the set-union of $R(x)$ and $R(y)$. In the case of a distributive lattice, the representations both by meet-irreducible and by completely meet-irreducible dual ideals are join-representations as well as meet-representations. In fact, any representation of a lattice by means of prime dual ideals is both a meet-representation and a join-representation, as will now be shown.

THEOREM 13. If $K$ is any collection of prime dual ideals of a lattice $L$, then the correspondence which assigns to each element $x$ of $L$ the set of all ideals of $K$ which contain $x$ is both a meet-representation and a join-representation of $L$.

Proof. By Theorem 10 the representation is a meet-representation. If $D$ is any dual ideal of $K$, then since $D$ is prime, it follows that $x$ or $y$ is in $D$ whenever $x \cup y$ is in $D$. Hence $D$ is in either $R(x)$ or $R(y)$ whenever $D$ is in $R(x \cup y)$. Conversely, if $D$ is in either $R(x)$ or $R(y)$, it is in $R(x \cup y)$ by definition of dual ideal. Therefore $R(x \cup y)$ is the set-union of $R(x)$ and $R(y)$, which was to be proved.

It should be noted that, although by Theorem 13 any representation of a lattice $L$ by prime dual ideals is both a meet-representation and a joinrepresentation, it cannot be an isomorphic representation unless the lattice $L$ is distributive, since the "ring of sets" on which $L$ is mapped by the representation is itself necessarily distributive.

9. Representations by maximal dual ideals. It was shown by Stone [1] that in Boolean algebras, which are distributive and relatively complemented lattices, prime ideals and maximal ideals are identical. In a merely distributive lattice, or in a lattice which is relatively complemented but not distributive, prime ideals and maximal ideals may not be identical. It has already been noted that distributive lattices may be represented by sets of prime ideals. Likewise, as we shall show, there are general classes of lattices, such as relatively complemented lattices, which may be represented by sets of maximal ideals. Neither kind of ideal, however, will suffice for the isomorphic 
representation of all lattices. For, on the one hand, the lattice of all real numbers has no maximal ideals. On the other hand, the lattice of all subspaces of a projective geometry with more than two points has no prime ideals.

For the representation of the general lattice by sets we have seen in Theorem 11 and its corollary that meet-irreducible and completely meetirreducible dual ideals suffice, and in Theorem 12 that these ideals specialize to prime ideals in distributive lattices. On the other hand, in many lattices which can be represented by sets of maximal ideals, the meet-irreducible and completely meet-irreducible dual ideals specialize to maximal dual ideals. But the situation is not so simple here, and we shall show by an example that there are cases where the representation of a lattice by maximal dual ideals is distinct from the representation of Theorem 11. But first we must consider the question of what lattices may be represented by sets of maximal dual ideals.

It was shown by Frink [2] that every complemented modular lattice may be isomorphically represented, with preservation of meets, by sets of maximal dual ideals. More generally, any relatively complemented lattice, whether modular or not, may be so represented. In fact, it will be shown that any lattice with the disjunction property has an isomorphic meet-representation by sets of maximal dual ideals. The proof of this, as well as the definition of the disjunction property, is due essentially to Wallman [1], although Wallman was mainly interested in the case of distributive lattices.

Definitions. A lattice $L$ with zero and unit elements $O$ and $I$ is said to be complemented if corresponding to every element $a$ of $L$ there exists an element $a^{\prime}$ of $L$ such that $a \cup a^{\prime}=I$, and $a \cap a^{\prime}=O$. The element $a^{\prime}$ is then said to be a complement of $a$.

A lattice $L$ is said to be relatively complemented if $a<b<c$ implies the existence of an element $b^{\prime}$ such that $b \cap b^{\prime}=a$, and $b \cup b^{\prime}=c$. The element $b^{\prime}$ is then said to be a complement of $b$ relative to the elements $a$ and $c$.

A lattice $L$ with $O$ element is said to have the disjunction property if whenever $a<b$, there exists an element $a^{\prime}$ such that $a \cap a^{\prime}=O$, and $a^{\prime} \cap b \neq O$.

It is easy to see that a relatively complemented lattice with $O$ element has the disjunction property. A fortiori, a complemented modular lattice, which is of necessity relatively complemented, has the disjunction property. We now show that lattices with the disjunction property may be represented by sets of maximal dual ideals.

THEOREM 14. If $L$ is a lattice with the disjunction property, then an isomorphic meet-representation of $L$ is obtained by assigning to each element $x$ of $L$ the set of all maximal dual ideals containing $x$.

Proof. By Theorem 10 it must be shown that if $x$ is distinct from $y$, then there exists a maximal dual ideal containing one but not the other of the pair $x, y$. Since $x$ is distinct from $y$, then either $x \cap y$ is distinct from $x$, or it is dis- 
tinct from $y$. Suppose the former; then $x \cap y<x$, and by the disjunction property, there exists an element $z$ of $L$ such that $x \cap y \cap z=O$, but $x \cap z \neq O$. By Zorn's lemma, there exists a maximal dual ideal containing $x \cap z$. This maximal dual ideal does not contain $y$, since then it would have to contain $x \cap y \cap z=O$, whence it would contain every element of $L$. Hence it contains $x$ but not $y$. Likewise if $z \cap y$ is distinct from $y$, there is a maximal dual ideal containing $y$ but not $x$. Hence by Theorem 10 , the representation of $L$ by sets of maximal dual ideals is an isomorphic meet-representation. This completes the proof.

We now wish to examine the relation between the representation of a lattice by sets of completely meet-irreducible dual ideals given in Theorem 11, and the representation by sets of maximal dual ideals just given in Theorem 14. We first note that by Corollary 1 to Theorem 8 , a dual ideal is completely meet-irreducible if and only if it is maximal subject to failure to contain some element of the lattice. Since, in a lattice with $O$ element, a maximal dual ideal is maximal subject to failure to contain the zero element $O$, we see that in such a lattice every maximal ideal is completely meet-irreducible. Conversely, in certain lattices with the disjunction property, such as the lattice of all linear subspaces of a projective geometry, it can be shown that every completely meet-irreducible dual ideal is maximal. Perhaps this is also true in every complete complemented modular lattice, although we have not yet been able to prove this. On the other hand, as we shall now show by an example, in some complemented modular lattices which are not complete, there exist completely meet-irreducible dual ideals which are not maximal. A fortiori, merely meet-irreducible dual ideals need not be maximal in complemented modular lattices, or in lattices with the disjunction property.

10. An example. Let $S$ be the projective coordinate space of countably infinite dimension, with coordinates taken to be the integers reduced modulo 2. (A general discussion of projective coordinate spaces will be found in the paper by Frink [2].) The points of $S$ are all infinite sequences $\left\{x_{n}\right\}$ of integers reduced modulo 2 such that $x_{n}=0$ except for a positive finite number of values of $n$. Collinearity and subspaces are defined in the usual way. The collection of all subspaces of $S$ is a complete complemented modular lattice.

Consider now the sublattice $L$ consisting of all subspaces of $S$ which are either of finite dimension, or are complements of subspaces of finite dimension. Note that although $L$ is a complemented modular lattice, it is not a complete lattice. Let $P$ denote the class of all elements of $L$ which are of infinite dimension, and $F$ the class of all elements of finite dimension. Although $F$ is a countable class, $P$ has the power of the continuum, since it contains a hyperplane corresponding to each sequence of integers reduced modulo 2 such as $\left\{y_{n}\right\}$, namely the subspace consisting of all points $\left\{x_{n}\right\}$ of $S$ such that $\sum x_{n} y_{n}=0$.

It is easily seen that $P$ is both a prime dual ideal of $L$ and a maximal dual 
ideal of $L$. We now construct a dual ideal of $L$ which is a proper subset of $P$ and which contains at least one complement of every element of $F$. To do so, select at random one complement of each element of $F$ to form a collection $K$. Extend $K$ to be a dual ideal $D$ by adjoining to it all superspaces of all finite intersections of elements of $K$. Since $K$ is a countable set, it can be seen that $D$ is also a countable set. Hence $D$ is a proper subset of $P$, since $P$ is not countable.

Let $A$ be an element of $P$ which is not in $D$. Extend $D$ to be a dual ideal $M$ which is maximal relative to failure to contain $A$. This can be done by Zorn's lemma. Note that $M$ can contain no element of $F$, since it contains a complement of each element of $F$. Hence $M$ is a proper subset of $P$. By Corollary 1 to Theorem $8, M$ is completely meet-irreducible, but $M$ is not maximal, since it is contained in the larger dual ideal $P$. We have thus exhibited in the complemented modular lattice $L$ a completely meet-irreducible dual ideal which is not maximal. It follows that the representation of a lattice given by Theorem 11 is in general distinct from that given by Theorem 14 . In lattices with the disjunction property, the representation by maximal dual ideals is in general more economical than that involving completely meet-irreducible dual ideals.

Incidentally, the example just constructed answers a question raised in the paper by Frink [2]. If a complemented modular lattice is indecomposable, is the projective space which it determines necessarily irreducible? The answer is in the negative, since the lattice $L$ of the example is indecomposable, but the projective space determined by it is reducible, since lines containing the dual ideal $P$ as a point are degenerate, and consist of just two points.

11. Preservation of arbitrary meets and joins. We shall call a dual ideal $D$ of a lattice $L$ a complete dual ideal if it contains the meet $\cap x_{a}$ of an arbitrary set $\left\{x_{a}\right\}$ of its elements whenever this meet exists in $L$. This is related to the notion of a completely prime dual ideal, defined in $\$ 6$. In fact it can be seen that a dual ideal in a lattice $L$ is completely prime if and only if its complement is a complete dual ideal in the lattice dual to $L$. In terms of these notions it is possible to state conditions that a representation of a lattice by sets of dual ideals shall preserve arbitrary meets or arbitrary joins of elements when these exist.

THEOREM 15. The meet-representation of a lattice $L$ which assigns to each element $x$ of $L$ the set of all dual ideals of a collection $K$ which contain $x$ preserves arbitrary meets of elements of $L$ if and only if all the dual ideals of $K$ are complete dual ideals, and preserves arbitrary joins if and only if all the ideals of $K$ are completely prime.

Proof. Suppose $x=\bigcap x_{a}$, and let $R(x)$ and $R\left(x_{a}\right)$ be the representative sets of $x$ and $x_{a}$ in the representation. To say that arbitrary meets are preserved is to say that $R(x)$ is the set-intersection of the set $R\left(x_{a}\right)$, that is, that a dual 
ideal $D$ of $K$ is in $R(x)$ if and only if it is in the sets $R\left(x_{a}\right)$. Since $D$ is in $R\left(x_{a}\right)$ if and only if $x_{a}$ is in $D$, this means that $x$ is in $D$ if and only if each $x_{a}$ is in $D$. But this is to say that $D$ is a complete dual ideal.

Now let $x=U x_{a}$. To say that the representation preserves arbitrary joins is to say that $R(x)$ is the set-union of the sets $R\left(x_{a}\right)$, which is to say that a dual ideal $D$ is in $R(x)$ if and only if it is in least one of the sets $R\left(x_{a}\right)$. But this means that $x$ is in $D$ if and only if at least one of the elements $x_{a}$ is in $D$, or in other words, if and only if $D$ is completely prime. In either case, therefore, the collection must consist entirely of dual ideals which are complete, or completely prime. This completes the proof.

It is easily seen that every principal ideal is complete. Conversely, in a complete lattice, every complete ideal is principal, since the meet $x$ of all elements of $D$ must exist and be a member of $D$. Hence $D$ must be the principal ideal $\{x\}$. We have, therefore, the following theorem.

THEOREM 16. Representations of a lattice $L$ by sets of principal dual ideals preserve arbitrary meets of elements of $L$. Conversely, if $L$ is a complete lattice, then the representations by principal dual ideals are the only representations which preserve arbitrary meets.

It is more difficult to discover significant isomorphic meet-representations which preserve arbitrary joins. Some rather strong condition must hold in order that enough completely prime dual ideals may exist to give an isomorphic representation.

In a distributive lattice, meet-irreducible dual ideals are prime by Theorem 12. It might be thought that similarly, in a lattice satisfying some infinite distributive law, such as $x \cap U y_{a}=\mathrm{U}\left(x \cap y_{a}\right)$, completely irreducible dual ideals are completely prime. If this were so, then the isomorphic representation of Theorem 11, using completely meet-irreducible dual ideals, would preserve arbitrary joins in such a lattice.

But consider the following counter-example. Let $L$ be the lattice of all finite subsets of an infinite set, together with their complements. Then $L$ is a Boolean algebra, and so it obeys all the usual infinite distributive laws. The collection $D$ of all elements of $L$ which are infinite sets is a maximal dual ideal of $L$, and is hence completely meet-irreducible. But $D$ is not completely prime, since it contains joins of finite sets without containing any finite sets.

The lattice $L$ of this example is not complete, and the question remains open whether, in a complete lattice satisf ying some infinite distributive law, completely meet-irreducible dual ideals are completely prime. It can be shown that this conjecture is verified for the case of complete atomic Boolean algebras. Whether it holds in general is an important unsolved problem concerning representations of the type of Theorem 11.

Another important unsolved problem of a similar nature concerns the possibility of isomorphic representations which preserve meets and joins of count- 
ably many elements in lattices in which such countable meets and joins exist. For example, the question whether the Boolean algebra $M / N$ of measurable sets modulo null sets has an isomorphic representation by sets which preserves countable meets and joins is of considerable importance in the theory of probability $\left({ }^{9}\right)$. The type of dual ideal that is required for such a representation is easily found by modifying the argument used in the proof of Theorem 15. Instead of complete dual ideals and completely prime dual ideals, it is necessary to use $\sigma$-complete and $\sigma$-prime dual ideals. The definitions of these differ from the definition of complete and completely prime dual ideals only in that they involve countable meets and joins, instead of arbitrary meets and joins. The principal difficulty is to determine whether enough of these ideals exist in a given lattice to furnish an isomorphic representation.

12. Representations by sets of lattice elements. In Theorem 16 and in the corollary to Theorem 10 we have considered meet-representations by sets of principal dual ideals. Since a principal dual ideal $\{x\}$ is completely determined by the element $x$ and conversely, we might just as well have considered meetrepresentations of a lattice by sets of elements of the lattice, rather than by sets of dual ideals. A. D. Campbell [1] has, in particular, studied meet-representations of lattices by sets of lattice elements of a special kind, namely joinirreducible elements. We now point out the relation of our results to his.

Recall from $\$ 3$ that an element $a$ of a lattice $L$ is said to be join-irreducible if it is not the join of two elements each distinct from $a$, and is said to be completely join-irreducible if it is not the join of any number of elements of $L$ each distinct from $a$.

THEOREM 17. A principal dual ideal $\{x\}$ of a lattice is meet-irreducible if and only if the element $x$ is join-irreducible. Likewise $\{x\}$ is completely meetirreducible if and only if $x$ is completely join-irreducible.

Proof. Suppose $x$ is not join-irreducible; then $x=y \cup_{z}$, where $y$ and $z$ are each distinct from $x$. Then the principal dual ideal $\{x\}$ is the set-intersection of the principal dual ideals $\{y\}$ and $\{z\}$. Hence $\{x\}$ is not meet-irreducible. Likewise if $x=\bigcup x_{a}$, where the elements $x_{a}$ are all distinct from $x$, then $\{x\}$ is the set-intersection of the distinct principal dual ideals $\left\{x_{a}\right\}$, whence $\{x\}$ is not completely meet-irreducible.

Conversely, suppose the principal dual ideal $\{x\}$ is the set-intersection of the dual ideals $D$ and $E$, each distinct from $\{x\}$. Then $D$ and $E$ contain elements $d$ and $e$ not in $\{x\}$. Hence $d \cap x$ is distinct from $x$, since otherwise $d \geqq x$, and $d$ would be in $\{x\}$. Likewise $e \cap x$ is distinct from $x$. But it is easily seen that $x=(d \cap x) \cup(e \cap x)$, whence $x$ is not join-irreducible. For, on the one hand, $(d \cap x) \cup(e \cap x)$ is in $\{x\}$, since it is in $D$ and $E$, and $x$ is the set-inter-

(9) Here the answer is in the negative. See L. H. Loomis, On the representation of $\sigma$-complete Boolean algebras, Bull. Amer. Math. Soc. vol. 53 (1947) pp. 757-760. 
section of $D$ and $E$; whence $(d \cap x) \cup(e \cap x) \geqq x$. On the other hand $(d \cap x)$ $\cup(e \cap x) \leqq x$ in any lattice. It follows that if $x$ is join-irreducible, then $\{x\}$ is meet-irreducible.

Finally, suppose that $\{x\}$ is the set-intersection of the collection $\left\{D_{a}\right\}$ of dual ideals $D_{a}$ each distinct from $\{x\}$. Select from each $D_{a}$ an element $d_{a}$ such that $d_{a}<x$. This is seen to be possible, since we may take $d_{a}$ to be $e_{a} \cap x$, where $e_{a}$ is an element of $D_{a}$ which is not in $\{x\}$. Now consider the join $\bigcup d_{a}$. This join exists and is the element $x$. For on the one hand $x$ is an upper bound of the elements $d_{a}$. On the other hand, if $y$ is any other upper bound, then $y$ is in each dual ideal $D_{a}$ and hence in their intersection $\{x\}$. Therefore $y>x$, and consequently $x$ is the least upper bound or join of the elements $d_{a}$, which are all distinct from $x$. It follows that $x$ is not completely join-irreducible. This completes the proof.

Corresponding to the meet-representation of a lattice determined by any collection $K$ of principal dual ideals $\{x\}$, as given by the corollary to Theorem 10 , there is an equivalent representation by sets of the corresponding lattice elements $x$, since principal dual ideals correspond one-to-one with the elements which determine them. Hence we have the following further corollary to Theorem 10:

COROLlARY. Given any set $M$ of elements of a lattice $L$, a meet-representation of $L$ is obtained by assigning to each element $x$ of $L$ the set of all elements $y$ of $M$ such that $y \leqq x$. This representation is isomorphic if and only if whenever the elements $x$ and $z$ of $L$ are distinct, there exists an element $y$ of $M$ such that of the two statements $y \leqq x$ and $y \leqq z$, one is true and the other is false.

It is easily seen that all dual ideals of a lattice are principal if and only if the lattice satisfies the descending chain condition. In such a lattice, all representations by sets of dual ideals are essentially representations by sets of lattice elements. In particular, the representation of Theorem 11 is, by Theorem 17, essentially just the representation by completely join-irreducible elements. For the case of lattices of finite length, this representation has already been discussed by A. D. Campbell [1].

\section{BIBLIOGRAPHY}

G. BIRKHOFF

1. On the combination of subalgebras, Proc. Cambridge Philos. Soc. vol. 29 (1933) pp. 441464.

2. Lattice theory, Amer. Math. Soc. Colloquium Publications, vol. 25, New York, 1940.

3. Subdirect unions in universal algebra, Bull. Amer. Math. Soc. vol. 50 (1944) pp. 764768.

A. D. CAmpbell

1. Set-coordinates for lattices, Bull. Amer. Math. Soc. vol. 49 (1943) pp. 395-398.

R. P. DILWORTH

1. Ideals in Birkhoff lattices, Trans. Amer. Math. Soc. vol. 49 (1941) pp. 325-353. 
N. DUNFoRd and M. H. STONE

1. On the representation theorem for Boolean algebras, Revista Ciencias Lima vol. 43 (1941) pp. 447-453.

O. FRINK

1. Representations of Boolean algebras, Bull. Amer. Math. Soc. vol. 47 (1941) pp. 755-756.

2. Complemented modular lattices and projective spaces of infinite dimension, Trans. Amer. Math. Soc. vol. 60 (1946) pp. 452-467.

M. H. STONE

1. The theory of representations for Boolean algebras, Trans. Amer. Math. Soc. vol. 40 (1936) pp. 37-111.

2. Topological representations of distributive lattices and Brouwerian logics, Casopis pro Peštovani Mathematiky a Fysiky vol. 67 (1937) pp. 1-25.

H. WALLMAN

1. Lattices and topological spaces, Ann. of Math. (2) vol. 39 (1938) pp. 112-126.

HARVARD UNIVERSITY,

Cambridge, Mass.

The Pennsylvania State College,

State College, Pa. 